\title{
une diagraphie de perméabilité par méthode acoustique
}

\author{
permeability log with an acoustical method
}

\author{
$1^{\text {re }}$ Partie : \\ P. MORLIER et N.E. BOUNOUA
}

Laboratoire de Génie Civil de l'Université de Bordeaux I* (UA 867 du C.N.R.S.)

$2^{e}$ Partie:

F. LEBRETON

Géodéveloppement **

Rev. Franç. Géotech. n 45 , p.p. $37-50$ (octobre 1988)

\section{Résumé}

Les auteurs présentent une diagraphie de perméabilité fondée sur une signature du signal acoustique, l'indice $I_{c}$ mesuré sur les premières arrivées d'ondes longitudinales.

Les bases physiques de cette diagraphie sont explicitées dans une première partie puis on montre quelques exemples de forages où la diagraphie de $I_{\mathrm{c}}$ et ses dérivés se sont avérés favorablement utiles.

\section{Abstract}

A $\log$ of permeability is presented in this paper; this $\log$ is based upon a signing of the acoustic signal, called index $I_{c}$, which is measured on the first arrival of compressional waves.

Physical principles of this log are given in a first part; then different examples show that the $I_{c} \log$ and other derived logs are essential tools for the knowledge of reservoir rocks. 


\section{$1^{\text {re }}$ PARTIE}

L'absorption sélective des hautes fréquences d'une impulsion acoustique est attribuée de façon principale, dans la gamme des fréquences utilisées en diagraphie, à la mobilité du fluide dans les pores de la roche théorie de BIOT —. Sa mesure exige une analyse de FOURIER dont la mise en œuvre est difficile en diagraphie acoustique en raison de la relative étroitesse des bandes passantes des transducteurs utilisés pour l'émission et la réception des ondes et parce que l'onde de compression n'est pure que sur un temps assez court. Aussi s'est-on fréquemment attaché à la définition des signatures robustes et simples du signal temporel : l'indice de forme I de LEBRETON (1) et le temps de montée de GLADWIN et STACEY (2) seront évoqués dans cet article; des études récentes ont montré que, à lithologie constante, il existe une relation linéaire entre ces deux indices et l'inverse de la constante de dissipation spécifique $\mathrm{Q}$ de la roche.

Nous avons cherché à exploiter une méthode de diagraphie robuste, en nous refusant d'imaginer de nouveaux outils et en utilisant donc des sondes usuelles, mais résolument acoustique puisque la propagation d'ondes est une des façons les plus pratiques de créer un déplacement relatif du fluide occupant les vides de la roche réservoir par rapport au squelette solide, c'està-dire une percolation, et la théorie de BIOT (3) met bien en évidence, sur des roches théoriques, cet aspect.

D'autre part, on sait que dans un train d'ondes de sonde acoustique, composé successivement d'ondes longitudinales, transversales, de surface (STONELEY)... non séparées, il est assez difficile d'attribuer une nature mécanique à telle ou telle partie du signal; l'idée fut donc de travailler sur le début du signal où l'on est sûr d'être en présence d'ondes longitudinales; l'inconvénient est le faible rapport signal sur bruit qui est un obstacle à la bonne qualité des signatures recherchées.

\section{ATTÉNUATION DES ONDES ET SES MESURES}

La figure 1 représente l'évolution d'une impulsion acoustique au fur et à mesure de sa propagation dans un milieu dissipatif; son amplitude diminue exponentiellement et elle devient de plus en plus étalée: elle s'appauvrit en hautes fréquences.

L'amplitude de l'onde - plane - $\mathrm{A}(\mathrm{x}, \mathrm{f})$, à une distance $\mathrm{x}, \mathrm{f}$ étant la fréquence, peut s'écrire sous la forme:

$$
\left.A(x, f)=A\left(x_{0}, f\right) e^{-x\left(x-x_{0}\right.}\right)
$$

où $\mathrm{A}\left(\mathrm{x}_{0}, \mathrm{f}\right)$ est l'amplitude à l'origine $\mathrm{x}_{0}$;

$\alpha$ est le coefficient d'atténuation, fonction croissante de f.

Pour une bande fréquentielle limitée, l'absorption relative de l'énergie $(\Delta \mathrm{E} / \mathrm{E})$ est sensiblement constante quelque soit la fréquence :

$$
\frac{\Delta \mathrm{E}}{\mathrm{E}}=2 \pi / \mathrm{Q}
$$

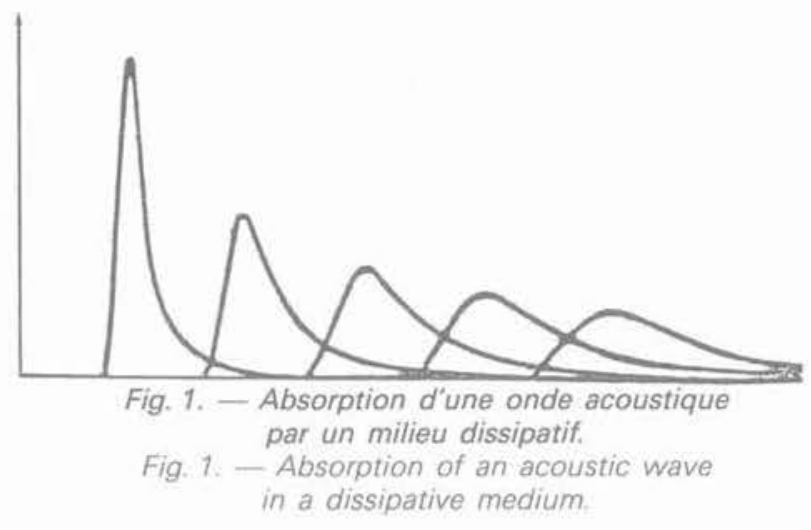

où Q, facteur de Qualité, est aussi appelé constante de dissipation spécifique.

Il est facile de montrer que $Q$ et $\alpha$ sont alors reliés par

$$
\alpha=\frac{\pi f}{Q V}
$$

où $\mathrm{V}$ est la vitesse de l'onde considérée.

\subsection{Indice de forme}

L'appauvrissement d'un signal de sonde acoustique classique en hautes fréquences peut se caractériser simplement par lamplitude comparée de la première alternance par rapport au niveau moyen du signal; c'est le sens de l'indice $I_{c}$ défini par LEBRETON (fig. 2).

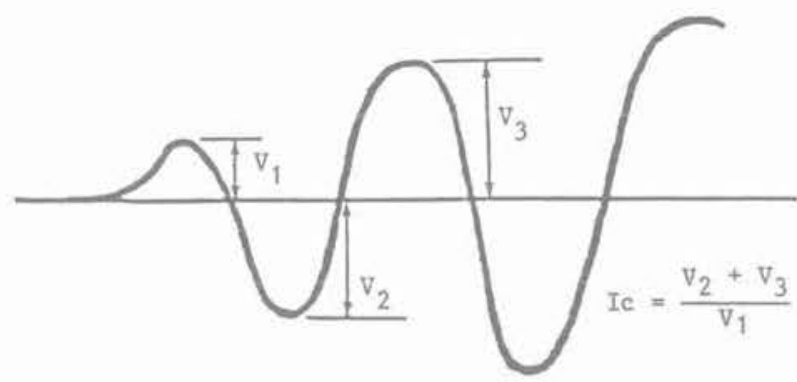

Fig. 2. - Définition de l'indice de forme $I_{c}$ (F. LEBRETON et al.).

Fig. 2. - Definition of Index $I_{c}$.

L'intérêt de cet indice est qu'il est indépendant du niveau général du signal dont on sait par ailleurs qu'il est notablement affaibli par la présence de cake (dépôt de boue à la paroi du forage); cet indice a également l'avantage d'être mesurable sur une sonde à un seul récepteur; sur une sonde normale, l'évolution de l'indice est, par contre, entachée d'erreurs par le bruit de fond, la linéarité des amplificateurs, l'interférence d'ondes non longitudinales sur les deuxième et troisième arches du signal.

L'étude numérique de ZHANG et al. (4) a montré (fig. 3) que, tout étant égal par ailleurs, en particulier l'impédance acoustique de la roche testée, l'indice Is varie linéairement avec $1 / \mathrm{Q}$. 


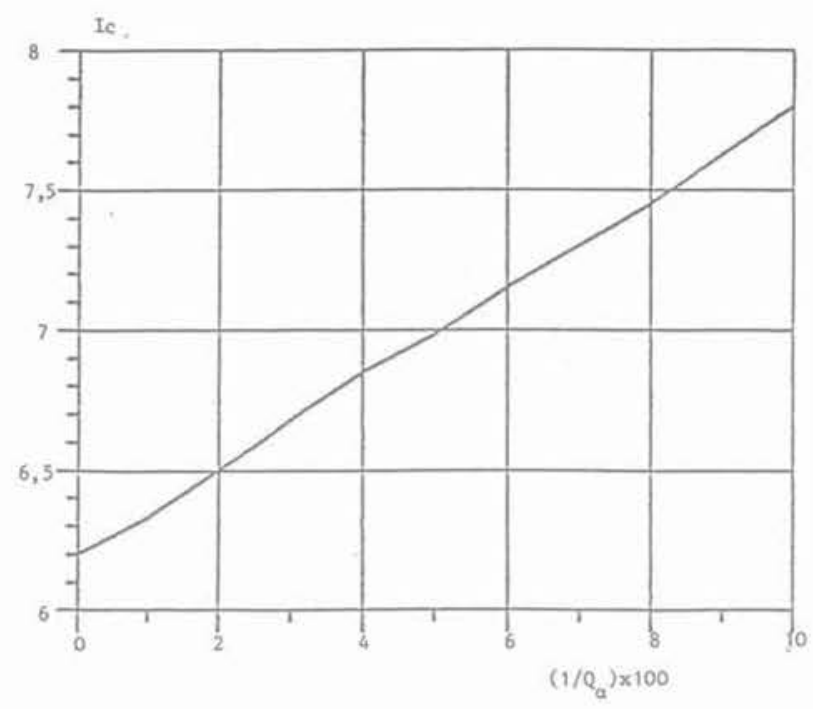

Fig. 3. - Relation entre l'indice de forme $I_{c}$ et la constante de dissipation $Q$, d'après ZHANG et al. Fig. 3. - Relation between Index $I_{0}$ and the quality factor $Q$, after ZHANG and al.

\subsection{Temps de montée}

Le même appauvrissement se traduit aussi simplement par un étalement progressif de la première arche du signal : GLADWIN et STACEY ont défini un temps de montée - (fig. 4) de façon rigoureuse; ils ont également

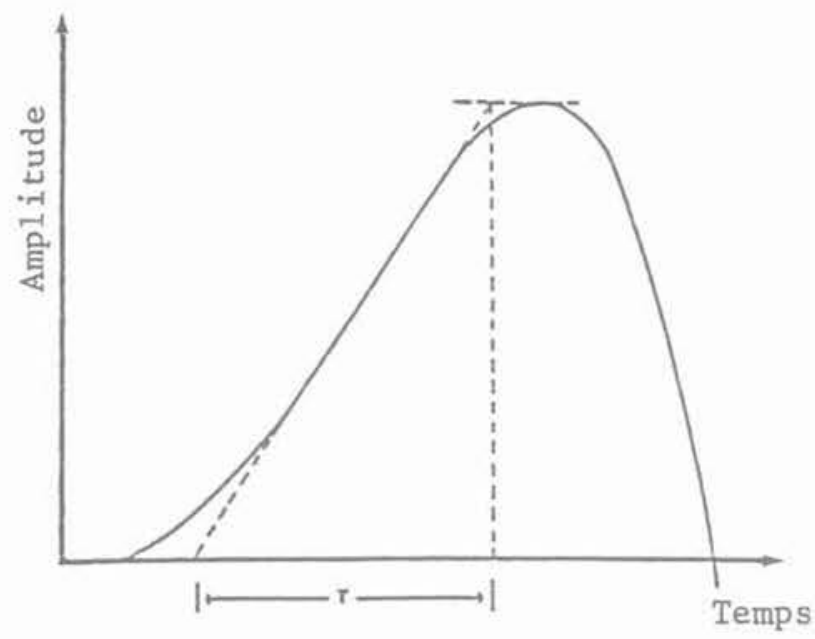

Fig. 4. - Définition du temps de montée (GLADWIN et al.).

Fig. 4. - Definition of rise time after GLADWIN and al.

montré que $\tau$ varie linéairement avec le rapport $t / Q$ ( $\mathrm{t}$ : temps de propagation):

$$
\tau=\tau_{0}+C t / Q
$$

où la constante $\mathrm{C}$ dépend essentiellement du type de source.

Cette étude a été reprise en France récemment au Laboratoire Central des Ponts et Chaussées par COTE et al. (5).
Indice $I_{c}$ et Temps de montée $\tau$ sont donc deux méthodes simples d'appréciation de l'atténuation des premières ondes acoustiques; leur mesure peut être quasiment normalisée ; on a vu au sujet de $I_{c}$, et c'est la même chose pour $\tau$, un certain nombre de raisons d'erreurs: niveau de bruit, pas d'échantillonnage, dynamique d'enregistrement pour l'essentiel.

Pour en finir sur ce point, on peut dire un mot de la profondeur d'investigation de telles diagraphies: des études effectuées sur un forage artificiel ont montré (1) que celle-ci peut être estimée à une demie longueur d'onde (environ dix centimètres).

\section{MÉCANISMES PHYSIQUES DE L'ATTÉNUATION}

Un certain nombre de mécanismes élémentaires peuvent expliquer l'atténuation des ondes de masse dans une roche, poreuse et saturée:

a. le frottement solide,

b. l'anélasticité de la matrice solide,

c. la diffraction de londe autour des grains,

d. la présence de bulles dans le fluide,

e. la relaxation visqueuse du fluide interstitiel,

f. le couplage fluide-solide de la théorie de BIOT,

g. le squirt ou squish flow, sorte de jaillissement du fluide au passage de l'onde mis en évidence par BOURBIE [6].

L'importance de chacun de ces mécanismes dépend de la pression effective sur la roche (a par exemple), de la pression du fluide (d ou e), de la température, de la fréquence, de la structure de la roche, c'est-à-dire de l'agencement de l'espace poreux d'où découle la perméabilité,

On imagine donc facilement qu'il est tentant de proposer des corrélations entre la perméabilité et l'atténuation des ondes de masse mais que la complexité de la structure des roches n'arrange pas les choses.

Nous avons effectué des études de laboratoire (7) ten. tant de mettre en évidence ces corrélations sur des grès pétroliers à la pression ordinaire; les résultats ont été les suivants :

Si l'on définit deux paramètres sans dimension:

$$
X=\frac{k f}{\mu \emptyset^{3}}
$$

k: perméabilité,

$f$ : fréquence de l'onde,

$\mu$ : viscosité du fluide mouillant,

$\emptyset$ : porosité,

et

$$
\mathrm{Y}=\frac{\alpha}{\mathrm{S}}
$$

$\alpha$ : atténuation (Neper/m),

$\mathrm{S}$ : surface spécifique de la roche $\left(\mathrm{m}^{-1}\right)$, 
alors que $\mathrm{X}$ varie sur six décades, $\mathrm{Y}$ suit une loi en $\mathrm{X}^{1 / 3}$ (fig. 5).

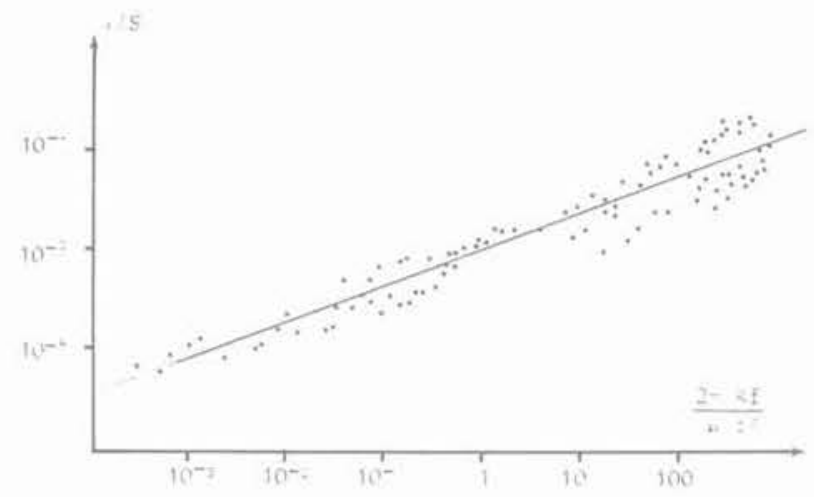

Fig. 5. - Atténuation a des ondes $P$ dans la roche en fonction de la fréquence $f$ de la perméabilité $k$ (7). Fig. 5. - Attenuation $\propto$ of $P$ waves in rock versus a dimensionless combination including frequency $f$ and permeability $k$.

Ces résultats montrent la complexité du rôle de la structure de la roche et qu'il sera donc difficile de faire de l'atténuation une «mesure » de la perméabilité ; par contre, il sera possible que, sur un réservoir donné, limité, l'évolution de la structure autorise de très bonnes corrélations entre $\alpha$ et $\mathrm{k}$.

\section{INDICE DE FORME ET PERMÉABILITÉ}

Une justification de la pertinence possible d'une corrélation indice de forme-perméabilité dont on comprend maintenant les raisons et les limites a été fournie par une campagne de mesures sur un forage artificiel pratiqué dans des bétons poreux (1): la porosité, constante, et la perméabilité y furent soigneusement et directernent mesurées; l'indice I fut relevé sur les mêmes volumes de quelques $\mathrm{dm}^{3}$ tout au long du forage, avec plusieurs types de sondes ; une corrélation, excellente au sens des diagraphies $(r=0,74)$ fut obtenue (fig. 6) entre $\mathrm{I}_{c}$ et $\mathrm{k}$ :

$\alpha$ et $\beta$ étant deux constantes.

$$
I_{c}=\alpha \log k+\beta,
$$

A la lumière de cette expérience et de campagnes ultérieures, on peut dire qu'une étude statistique de l'incide $I_{c}$ dans un réservoir a de fortes chances de mettre en évidence les zones perméables et même de donner une bonne évaluation de la perméabilité si celleci est de même nature sur tout le réservoir.

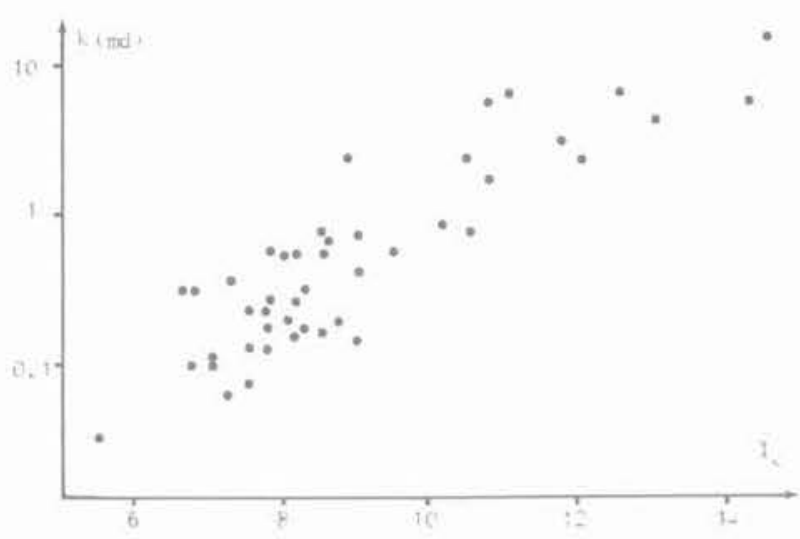

Fig. 6. - Relation perméabilité $k$-indice $I_{c}(1)$. Fig. 6. - Permeability $k$ versus Index $l_{c}$.

\section{BIBLIOGRAPHIE}

1. LEBRETON F., MORLIER P. (1983), Une diagraphie de perméabilité par méthode acoustique, Bulletin de l'Association Internationale de Géologie de l'Ingénieur $\mathrm{n}^{\circ}$ 26-27, Paris, 1983.

2. GLADWIN M.T., STACEY F.D. (1974), Anelastic degradation of acoustic pulses in rock, Phys. Earth Planet. Inter., 8, 332-336, 1974.

3. BIOT M.A. (1956), Theory of propagation of elastic waves in a fluid-saturated porous solid I. lower frequency range; II. higher frequency range, J. Acoust. Soc. Am., 28, 1956 a : $168-178,1956$ b: 179-191.

4. ZHANG J. et CHENG C.H. (1984), Numerical studies of body wave amplitude in full waveform acoustic logs, Neuvième Colloque International de Diagraphies, oct. 1984.

5. COTE Ph., CAMPILLO M. (1985), Propagation en milieux fissurés. Mesures in-situ du facteur de qua. lité et des vitesses sismiques, Rapport A.T.P. 1985.

6. BOURBIE T., COUSSY D. (1986), Acoustique des milieux porewx, Edition Technip, 1986.

7. MORLIER P., SARDA J.P. (1971), Atténuation des ondes élastiques dans les roches poreuses saturées, A.R.T.E.P., 1971. 


\section{$2^{e}$ PARTIE}

L'étude théorique a fait apparaître les propriêtés du rapport I (ou de tout indice dérivé de celui-ci) pour la mesure de la mobilité $(\mathrm{k} / \mu)$ du fluide dans le terrain traversé par le signal. L'analyse de l'évolution diagraphique de la perméabilité (Darcilog) * est développée ci-après.

\section{CARACTÉRISTIQUES DE L'INDICE $I_{c}$}

1.1. Aucune loi de composition des mesures directes de perméabilité faites sur échantillons prélevés sur un plus gros volume de milieu poreux naturel ne permet de retrouver à coup sûr la mesure directe de perméabilité initialement faite sur ce gros volume.

Il en résulte que, pour qu'elle ait une signification, toute mesure directe ou indirecte de perméabilité sur un milieu poreux doit être faite sur un volume et selon une direction déterminés.

Dans un forage la mesure de I est faite à partir d'un ensemble Emetteur-Récepteur (E-R) : son volume d'investigation est celui qui est donné par l'outil dont l'écart $E-R$ est de 3 à 5 fois la longueur d'onde du signal émis (1) (2).

Comme les transducteurs des sondes acoustiques actuelles ne présentent pas de symétrie axiale de révolu. tion, ils doivent être excentrés judicieusement dans les forages (3).

Il faut noter que cette mesure acoustique est effectuée in-situ dans un milieu continu, semi-infini puisque limité seulement par un secteur de cylindre du puits. En revanche, les mesures acoustiques ou d'écoulement de fluide sur carottes sont influencées par des limites géométriques imposées dans toutes les directions.

Le volume utile d'investigation de l'indice 1 (fréquence de 12 à $30 \mathrm{KH}$ ) est alors celui d'un secteur circulaire de 10 à $20 \mathrm{~cm}$ de pénétration radiale, de 15 à $30 \mathrm{~cm}$ de hauteur, centré sur le milieu du segment ER. La perméabilité y est mesurée suivant la direction de l'axe du forage.

Dans un puits les mesures successives de la diagraphie 1. doivent donc être espacées d'environ $15 \mathrm{~cm}$ pour que toute la hauteur du découvert soit correctement investiguée.

1.2. Le signal émis provoque toujours une dépression initiale qui, après passage dans le milieu poreux, se retrouve au début de l'onde $\mathrm{P}$ du signal reçu comme une amplitude (négative) toujours faible devant les deux suivantes.

Pour que la mesure I reste précise la puissance du signal émis doit donc ếtre d'abord suffisante.

La vitesse de remontée de l'outil dans le forage agit directement sur l'amplitude du bruit surimposé au signal proprement dit. Pour réduire cet effet de bruit la vitesse doit être faible (inférieure ou égale à $300 \mathrm{~m} /$ heure) et régulière. Des vitesses de remontée aussi basses que $100 \mathrm{~m}$ /heure peuvent se révéler nécessaires.

Par ailleurs le gain choisi pour l'enregistrement doit satisfaire aux deux conditions antagonistes suivantes:

- obtenir une amplitude décelable, chiffrée sur la première dépression mesurée

- éviter la saturation des autres amplitudes de l'onde $\mathrm{P}$ reçue.

Le pas d'échantillonnage du signal doit être au plus le vingtième de la période du signal, soit pour une fréquence de $20 \mathrm{HKz}$, une prise d'informations toutes les 2 us.

Dans un puits la suite des valeurs de I relevées en fonction de la profondeur permet d'établir la diagraphie I de base (Darcilog). Avec les outils acoustiques actuels la gamme de variation de I s'étend de 2 à 200 environ, selon les perméabilités rencontrées.

\section{CALIBRATION $I_{c}=f(k)$}

Toutes les fois que mesures directes de perméabilité et mesures de I sont faites au droit d'un même intervalle dans un puits, la comparaison de ces deux séries de mesures conduit à proposer une relation:

$$
I_{c}=\alpha \log \frac{k}{\mu}+\beta
$$

où $\alpha$ et $\beta$ dépendent de la position des transducteurs dans l'outil et du centrage de l'outil dans le forage.

A ce jour (1988), la détermination de $\alpha$ et $\beta$ n'a pas été publiée et il ne sera pas fait état ici des valeurs attribuables à ces paramètres.

\section{VISCOSITÉ}

C'est en général un mélange d'eau connée, de filtrat de boue avec des traces de gaz plus ou moins dissous qui sature la zone d'investigation de la mesure $I_{c}$ dans les puits.

La valeur de la viscosité dynamique de ce mélange varie en général dans des limites assez étroites pour que l'ordre de grandeur de la mobilité dans les roches n'en soit pas modifié.

En revanche dans la zone investiguée la présence de saturation en hydrocarbures (liquides ou gaz) a une influence lisible sur I. L'emploi successif de deux fré. quences d'émission du signal, et donc de deux rayons d'investigation pour $\mathrm{I}$, permet le repérage de l'existence d'une zone envahie par un filtrat de viscosité très différente de celle du fluide initial.

\footnotetext{
* Marque déposée.
} 


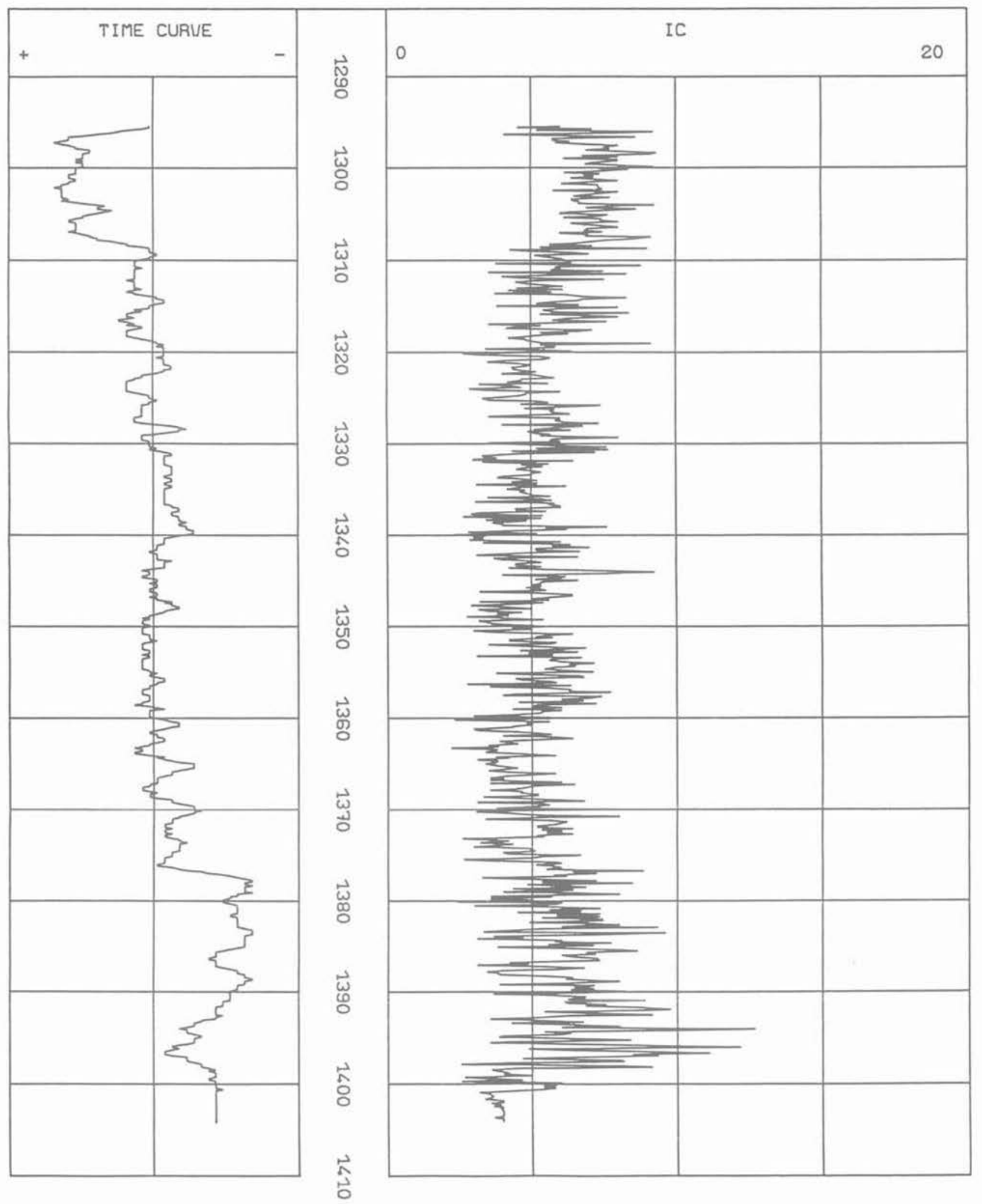

Fig. 7,7 bis, 7 ter. - L'histogramme des valeurs de $I_{c}$ pour cet intervalle d'une centaine de mètres montre une majorité de faibles valeurs de $I_{\mathrm{c}}$ (roche imperméable); pour la zone 1392-1398, les valeurs élevées de $I_{\mathrm{c}}$ suggèrent des fractures ouvertes mais le log de débitmétrie n'a pas été effectué.

Fig. 7. 7 bis. 7 ter. - The $I_{c}$ value histogram built for this 100 -meter thick interval makes clear it is made up of low $I_{\mathrm{c}}$ values, i.e. impervious rocks, but over 6 meters $(1392.1398 \mathrm{~m})$. Over these 6 meters some high values for $I_{\mathrm{c}}$ suggest open fractures apt to provoke a flow, but the spinner flowmeter log was not run. 


\section{AUTRES DIAGRAPHIES : EXAFLO * ET SEALDEX}

Sur un découvert donné, l'histogramme des I présente des modes dont les valeurs représentatives correspondent à des familles de perméabilité aptes à êtres reconnus sur déblais, carottes, tests, diagraphie ou global ". Ainsi les valeurs de I élevées ( $>50$ par exemple pour l'outil B.H.C.) et isolées sont normalement indicatives de passées fracturées.

Ainsi au droit des roches non fracturées à matrices imperméables (ex. : argiles) $I_{c}$, mesuré avec le B.H.C., est compris entre 6 et 10 .

Sur un intervalle sédimentaire épais (ex.: 50 à $100 \mathrm{~m}$ ), les passées imperméables sont souvent prépondérantes: 15 à $30 \%$ de l'ensemble et cette proportion se retrouve évidemment sur l'histogramme de $I_{c}$, par exemple (fig. 7).

La basse valeur modale de ces passées imperméables, relevée sur l'histogramme est en fait une valeur de coupure séparant les bancs susceptibles de production ou d'injection de fluides aux environs de la pression hydrostatique correspondante.

\section{CUMUL DE LA PERMÉABILITÉ CALCULÉE OU EXAFLO *}

Entre deux cotes de profondeur dans un forage, le cumul de la perméabilité verticale déduite de I par la formule précitée constitue une nouvelle diagraphie dont les informations peuvent, sous certaines conditions, reconstituer celles de la débitmétrie enregistrée avec une sonde à micromoulinet en découvert.

Toutes les valeurs de $I_{c}$ inférieures à la coupure $\left(I_{c}=7\right.$ dans l'exemple de la figure 7) sont éliminées du cumul puisqu'elles sont relatives à des passées non-susceptibles d'écoulement.

En revanche sì même une seule valeur de I n'a pas été calculée à une cote donnée à cause, par exemple, d'un signal reçu de mauvaise qualité, le cumul n'est valable qu'au-dessus ou au-dessous de cette cote.

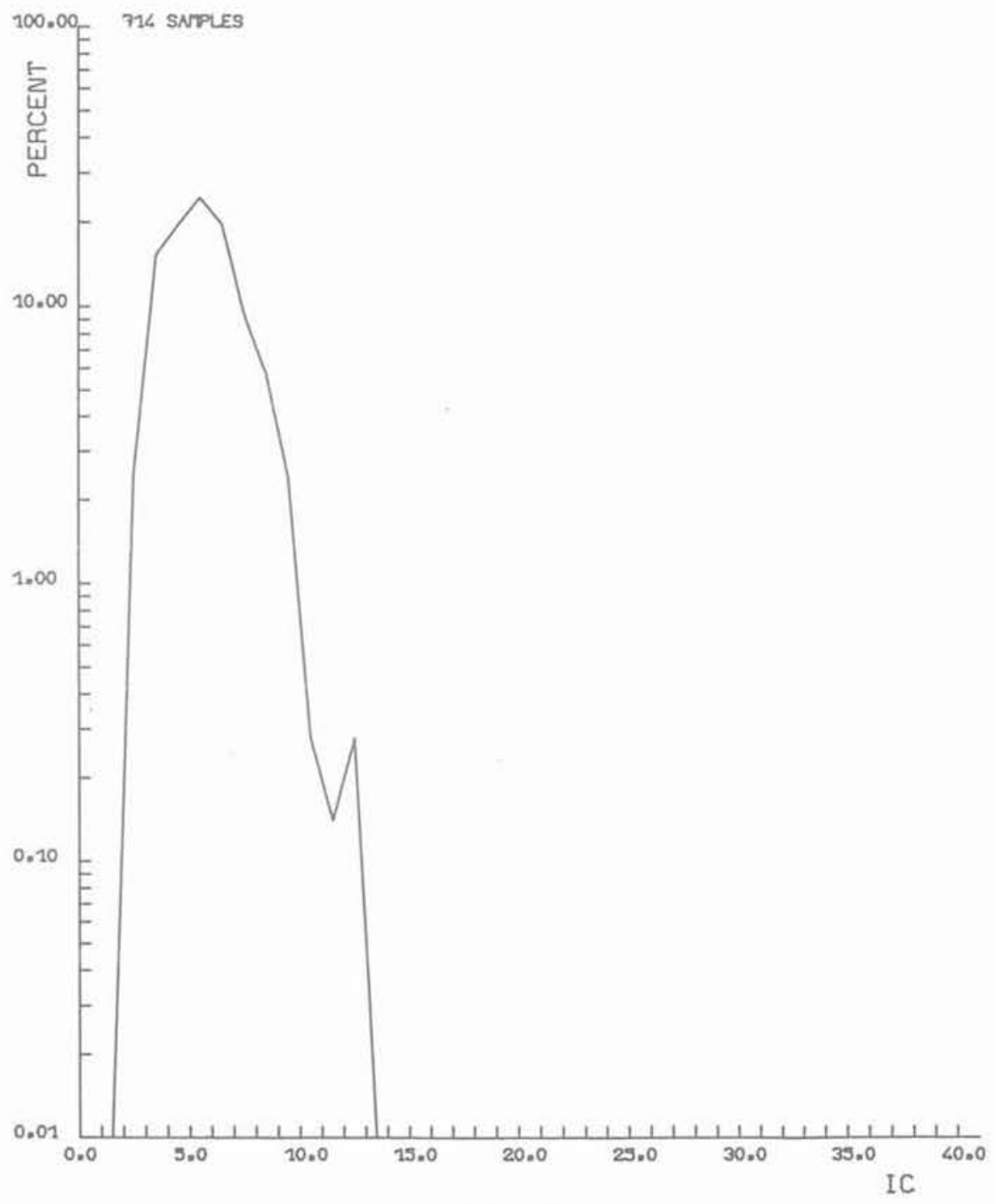

Fig. 7 bis 


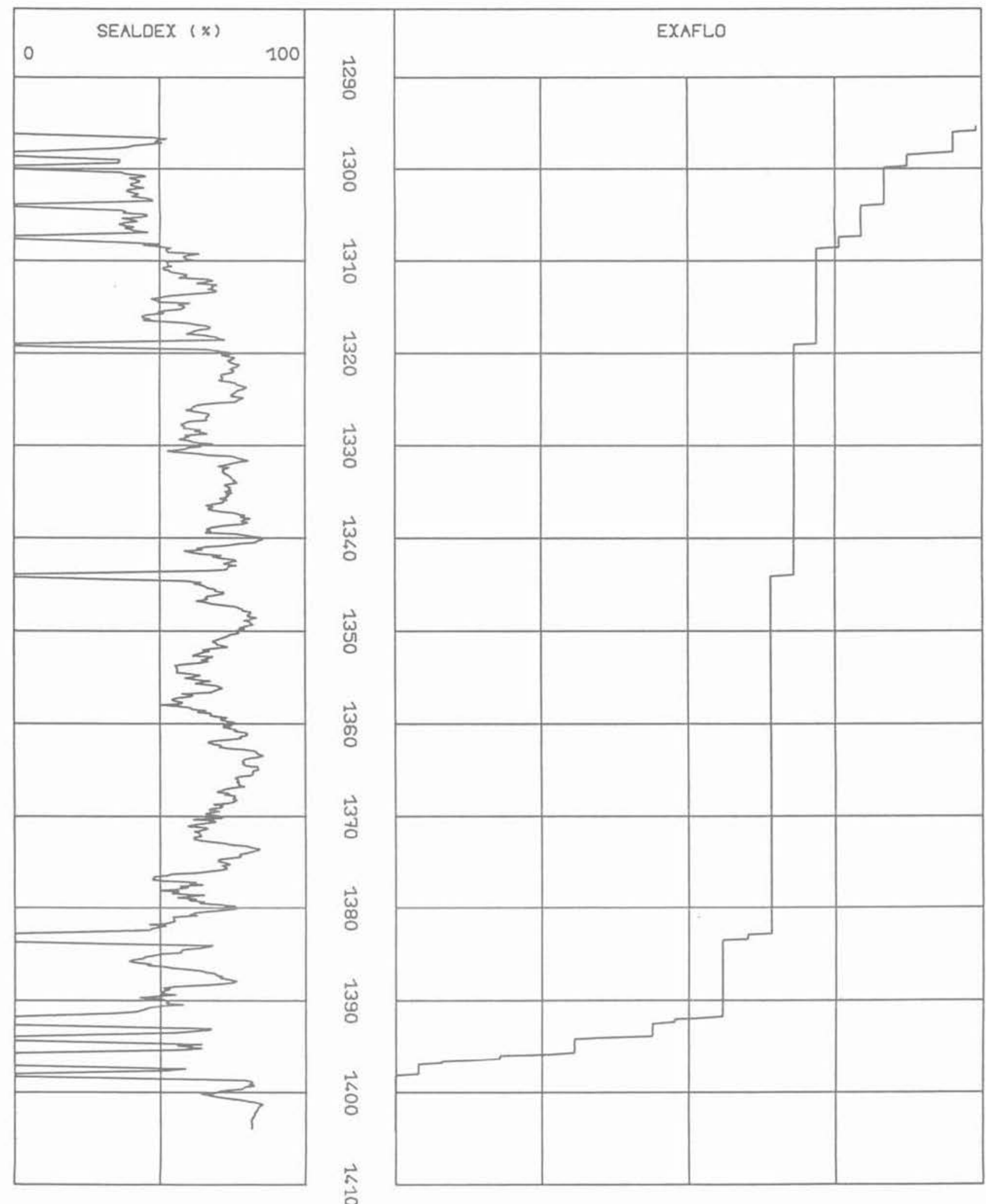

Fig. 7 ter

Les comparaisons entre EXAFLO ${ }^{*}$ et diagraphie de débitmétrie sont possibles toutes les fois que les deux diagraphies sont faites en découvert sur un même puits. Une bonne corrélation entre les deux diagrammes est attendue et généralement trouvée, mais des écarts sont aussi parfois notés. Ces écarts entre le profil de l'Exaflo * d'une part et de la débitmétrie d'autre part sont inter- prétables en termes de pression de gisement différente de la pression hydrostatique et/ou de variation de la viscosité du fluide saturant, avec la profondeur (fig. 7 bis).

Historiquement les deux premiers exemples d'une telle comparaison datent de 1983. Ils proviennent de deux 

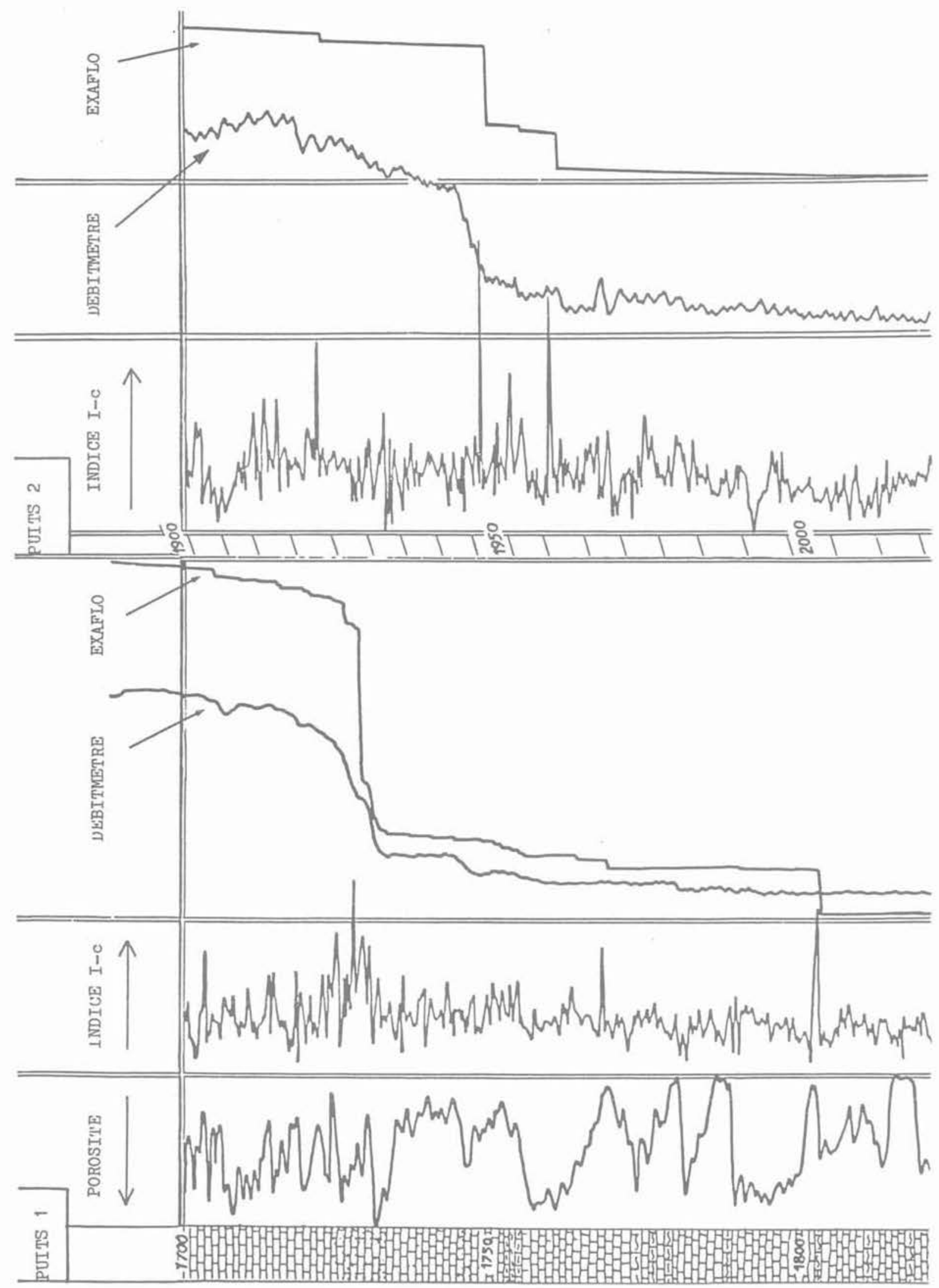

Fig. 8. - Sur un aquifère géothermal calcaire épais de $120 \mathrm{~m}$, deux puits ont été forés à 1 mile de distance; EXAFLO et le log de débitmétrie ont été effectués sur chacun de ces puits.

La superposition de EXAFLO et du débitmètre donne des corrélations satisfaisantes dans les deux cas.

Cette opération, effectuée en 1982, est historiquement le premier essai du nouveau log EXAFLO.

Fig. 8. - Over a 120-meter thick geothermal limestone aquifer two wells were drilled 1-mile apart. EXAFLO ${ }^{*}$ and the spinner flowmeter $\log$ were run in each of them.

Superposing EXAFLO and flowmeter profiles yields a satisfactory correlation in both cases. This glaring demonstration made in 1982 is historically the first try of the new log dubbed EXAFLO. 


\section{INAG III-9 COMPARAISON INDICE Ic,MICROMOULINET EN INJECTION,GRADIENT DE TEMPERATURE}
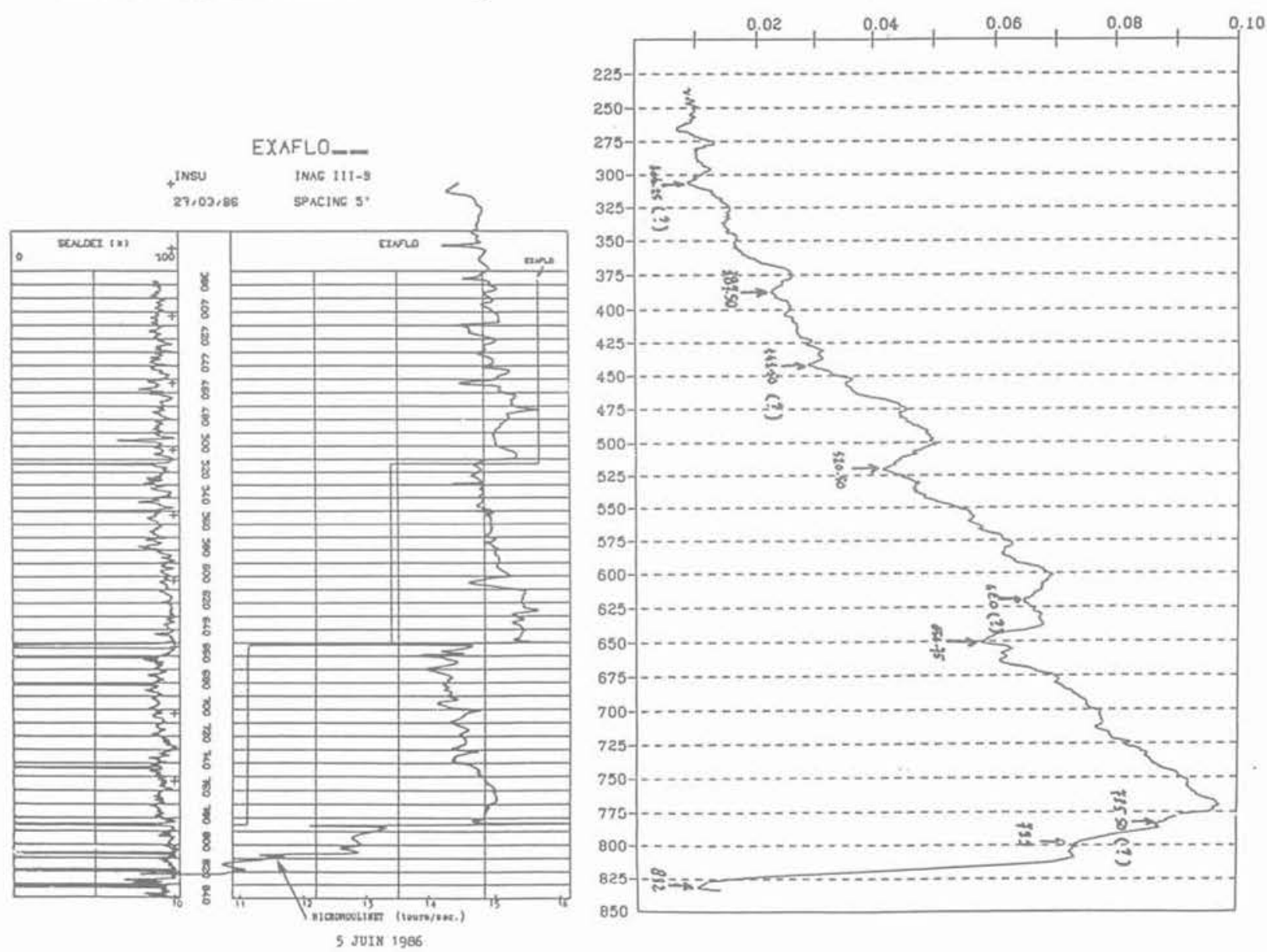

Fig. 9. - A travers un massif granitique dans le Massif Central, un puits de $840 \mathrm{~m}$ a été foré, trois logs différents ont été effectués pour détecter d'éventuelles venues d'eau: EXAFLO, log de température avec une précision de $+0,01{ }^{\circ} \mathrm{C}$, et le micromoulinet lors d'une injection d'eau à pression constante à partir de la surface.

Fig. 9. - Through a granite body lying in Central France, a 840 meter deep well was drilled and three different logs were tried in it for detecting water entries: EXAFLO: Temperature $\log \left( \pm 0.01^{\circ}\right.$ Celsius accuracy). and spinner flowmeter log run while water was injected at constant pressure from the surface.

puits de géothermie du B.R.G.M. (fig. 8), au droit des carbonates poreux fissurés, karstifiés, mais non fracturés du Dogger.

L'exemple le plus récent (1986) (fig. 9) est relatif à un puits foré dans le granite du Massif Central. Toutes les passées de granite qui absorbent des volumes notables d'eau injectée à pression constante sont signalées par la diagraphie de débitmétrie (micromoulinet) et par I'Exaflo:

Un regroupement supplémentaire de l'accord de ces deux mesures est fourni par la diagraphie des écarts de température enregistrée avec un thermomètre sensible au $1 / 100$ de degré, effectuée par Monsieur JOLIVET.

Un autre exemple (1986) est celui de terrains sableux deltaïques de plus de $300 \mathrm{~m}$ d'épaisseur, avec quelques minces passées argileuses.

A l'aplomb du forage (fig. 10 et 10 bis), la porosité des sables est comprise entre 30 et $40 \%$ : des tests de production infructueux ont permis de s'assurer que leur perméabilité est la plupart du temps trop faible pour assurer le débit d'eau souhaité $\left(500 \mathrm{~m}^{3} /\right.$ heure).

Le plus perméable des horizons vers $230-232 \mathrm{~m}$, est repéré grâce à l'Exaflo:. Un test de production a confirmé l'exactitude de cette information.

\section{SEALDEX}

Compte tenu de ce qu'elles sont très faibles, les perméabilités des intervalles de terrain représentées par des valeurs d'indice I inférieures à la coupure définie ci-dessus peuvent être composées selon plusieurs modes de calcul.

Il a semblé judicieux de les représenter par une simple moyenne des valeurs trouvées sur l'intervalle E-R, sous 


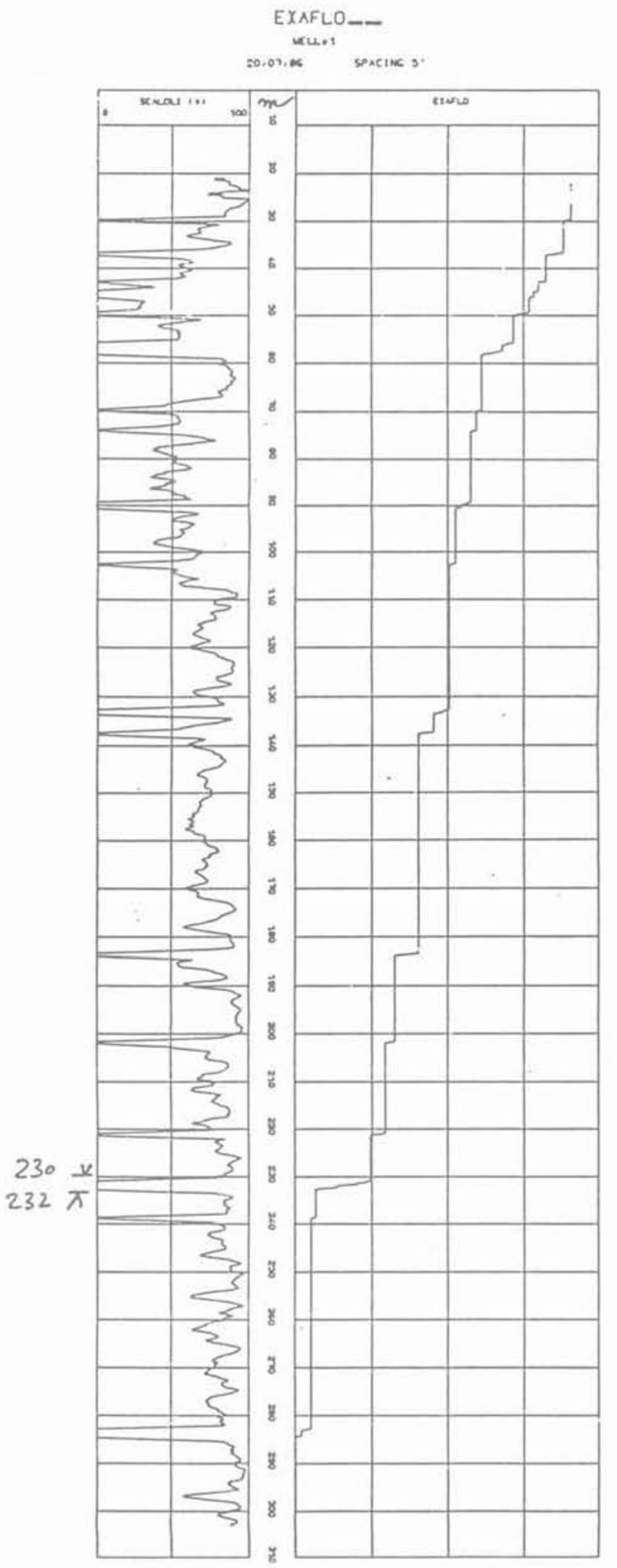

Fig. 10, 10 bis. - Dans ce puits de $300 \mathrm{~m}$, la résistivité, la polarisation spontanée et le log de radioactivité naturelle caractérisent un sable propre, saturé d'eau, avec une porosité de 0,30 à 0,40 . Aucun de ces logs ne peut mettre en évidence le meilleur intervalle de production.

Les logs $I_{c}$ et EXAFLO ont permis d'individualiser une zone perméable $(230-232 \mathrm{~m})$ qui produit $25 \%$ du débit total. Fig. 10, 10 bis. - In this 300-meter deep well Resistivity. SP. and Natural Gamma Ray logs show clean. $30 \%$ and $40 \%$ porous, fresh-water saturated sands.

All these logs are of no use to single out the best water producing interval.

Fortunately $l_{\mathrm{c}}$ and EXAFLO ${ }^{\circ}$ were also run and from them the 2-meter thick interval $(230-232 \mathrm{~m})$ was singled out and it produces $25 \%$ of the total flow. 


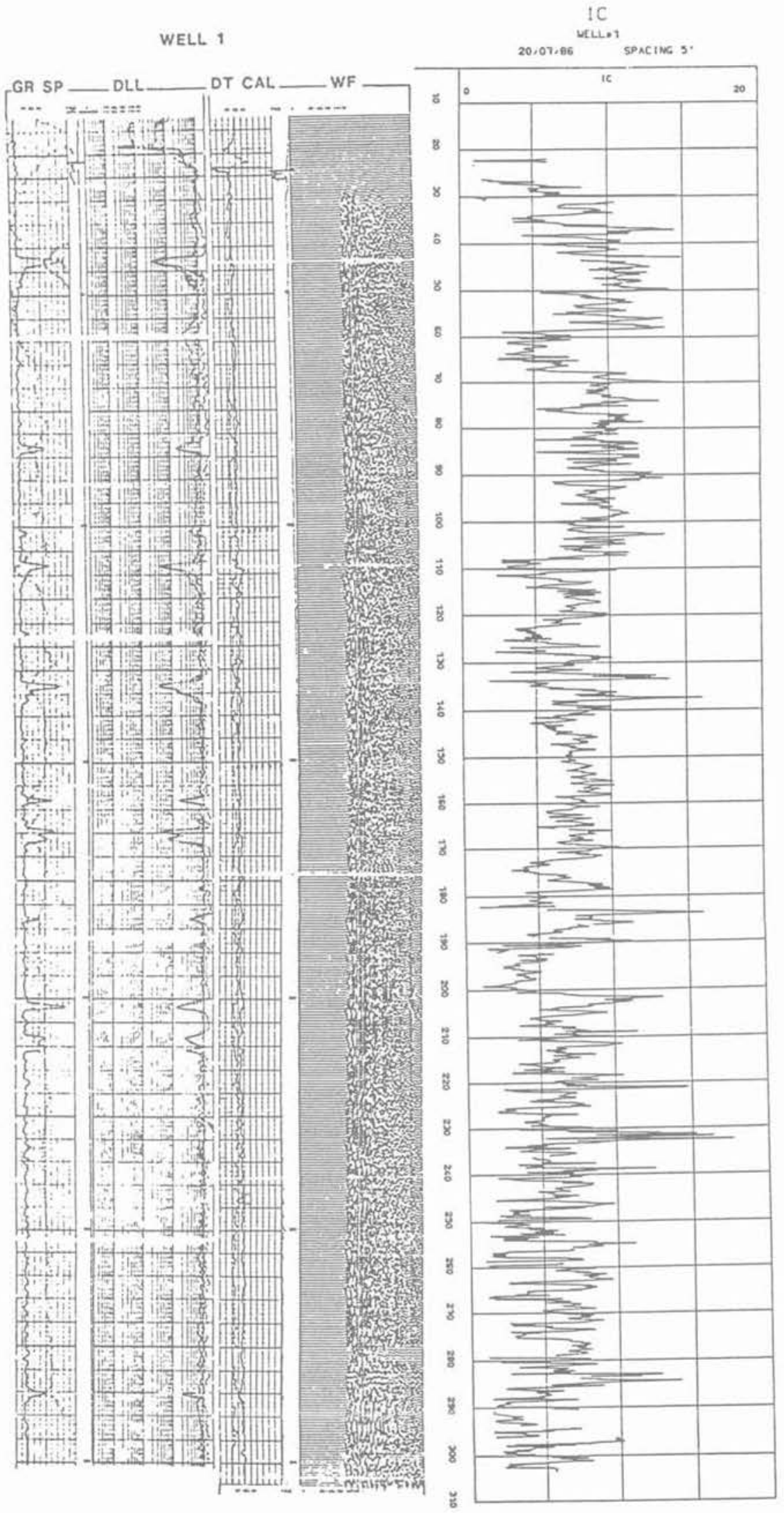

Fig. 10 bis 


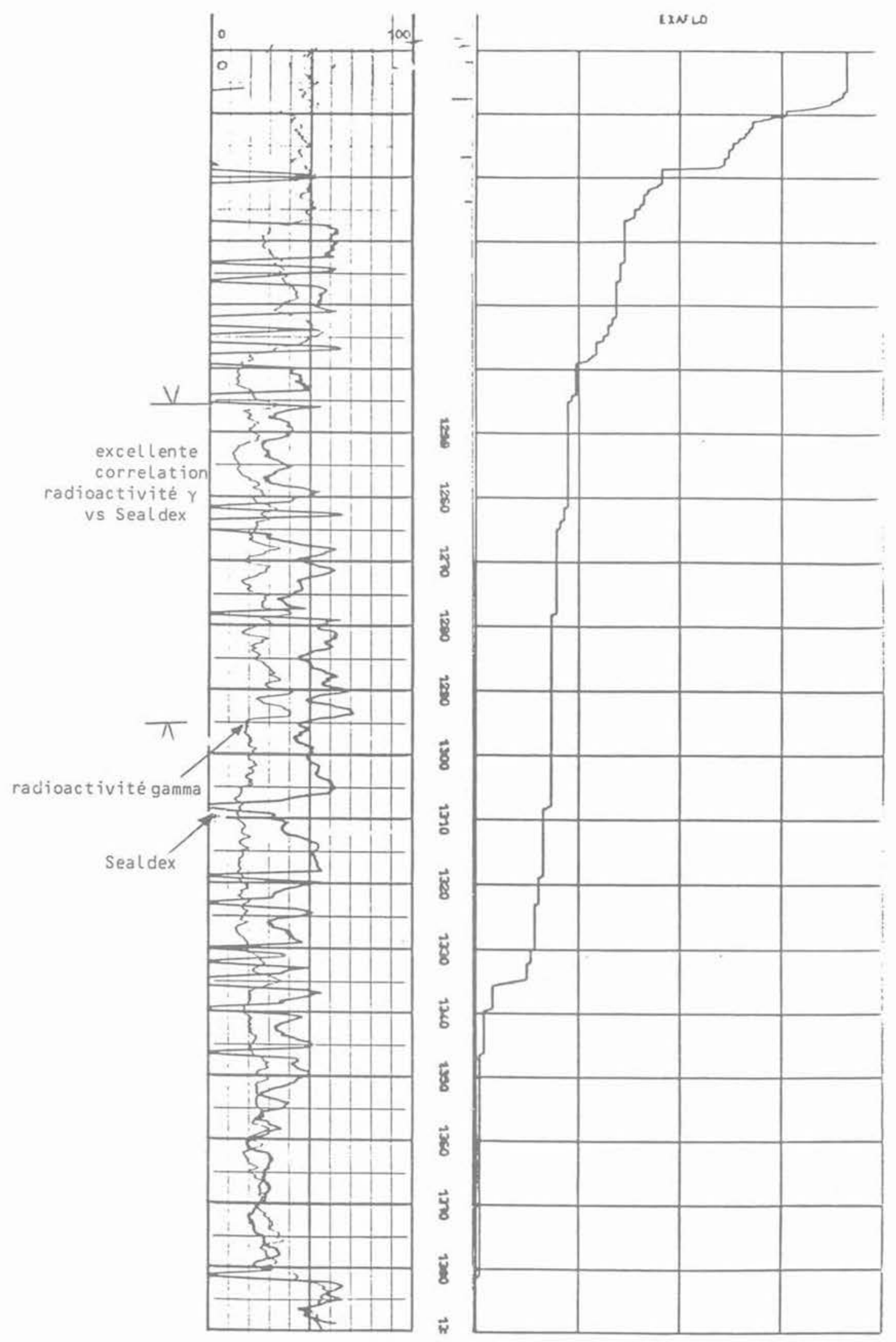

Fig. 11. - Dans ce puits, la formation est composée de grès plus ou moins argileux; la perméabilité de ce type de roche dépend de la granulométrie du sable et de la distribution de largile. Il peut donc arriver que, sur un intervalle à granulométrie constante, le seul pourcentage en argile soit responsable de l'imperméabilité.

Si c'est le cas, le log de radioactivité naturelle et le log $I_{c}$ (ou SEALDEX) doivent présenter un profil parallèle: on notera. de 1250 à $1300 \mathrm{~m}$, la remarquable identité des deux logs.

Fig. 11. - In this well the shown formation is made up of more or less shaly sandstone.

The permeability of this type of rock is controlled by sand granulometry and by clay distribution.

So in such rocks it may occur that over an interval of constant grain size the only impermeability agent be the given clay percentage.

In this is the case the Natural Radioactivity log and the $I_{c} \log$ (or "Sealdex". Which is the averaged $I_{c}$ over the spacing length) ought to exhibit identical profiles. From 1250 to $1300-\mathrm{m}$ identity of these two profiles is indeed striking. 
la condition qu'aucune d'entre elles n'y soit supérieure à la valeur de coupure.

Dans les terrains où la teneur en argile contrôle la perméabilité, le profil de l'information fourni par cette diagraphie Sealdex corrèlera avec la diagraphie de $\mathrm{Ra}$ dioactivité gamma qui mesure la teneur en argile (fig. 11).

\section{CONCLUSION}

Dans un puits la recherche des intervalles au droit desquels se produisent des écoulements de fluide entre roche et forage conduit à la recherche des intervalles perméables.

Bien entendu le débit de l'écoulement (et sa permanence) ne dépendent pas seulement de la valeur de la perméabilité sur cet intervalle. L'évaluation de la perméabilité par les diagraphies acoustiques présentées ici n'est donc qu'un élément d'enquête sur les écoule. ments.

En revanche pour l'emploi de la diagraphie acoustique par traitement de l'onde $\mathrm{P}$, il est urgent que la procédure de mesure soit entièrement définie: c'est ce qui a été fait plus haut.

A condition qu'ils aient été enregistrés suivant les règles de l'art, tous les exemples obtenus à ce jour ont donné des diagraphies fidèles. Leur interprétation en termes de $\mathrm{k} / \mu$ s'est toujours révélée utile à l'ingénieur.

\section{BIBLIOGRAPHIE}

1. LEBRETON et al. (1978), SPWLA Logging Symposium Transactions, 1978, El Paso.

2. CONCHE, LEBRETON et ROJAS (1986), International Geothermy Symposium, janv. 1986, Stanford.

3. Brevets: France $n^{\circ} 2106702$, U.S.A. $n^{\circ} 3786894$, Canada $n^{\circ} 852617$. 